and thus be cost-saving especially after HPV testing is deployed as a screening tool. However, it is in the post-vaccination era when the cohorts of women vaccinated in their teens enter screening age that this approach may prove most valuable by permitting a surveillance system that can serve two roles simultaneously: monitoring duration of vaccine protection (with HPV typing for those who are positive) and screening for cervical cancer. The author will present the arguments for an integrated approach that involve the two prevention strategies against this disease: HPV vaccination and molecular testing in cervical cancer screening.

\section{S12.3 WHAT IS THE LATEST DATA FROM THE 2 COMMERCIALLY AVAILABLE PROPHYLACTIC CERVICAL CANCER VACCINES?}

doi:10.1136/sextrans-2011-050102.50

S Garland. Royal Women's hospital, Victoria, Australia

Since 2007 two prophylactic HPV vaccines (a bivalent incorporating VLPs 16 and 18, plus a quadrivalent vaccine with VLPs 16, 18, 6, 11) have been licensed for use. This was based on excellent efficacy, immunogenicity and safety data from phase 3 clinical trials. In addition there is also some cross protection for disease for phylogenetically related genotypes. Where these vaccines have been incorporated into public health programs with high coverage, (particularly targeted school based programs), already reductions in those disease those with the shortest incubation periods, are being seen that is, genital warts for the quadrivalent vaccine.

The greatest challenge today is to obtain wide vaccine coverage to those countries with the highest incidence of disease.

\section{S12.4 GENITAL WARTS: PREVENTION, DIAGNOSIS, TREATMENT AND COUNSELLING}

doi:10.1136/sextrans-2011-050102.51

M Steben. Institut national de santé publique du Québec, Montréal, Canada

Genital warts (GW) are one of the most common reasons for consultation at an STI clinic. A majority of partners sexually exposed to GW will develop GW. Half of cases of GW will have cleared within 4 months. Condoms' efficacy has been demonstrated but need high compliance to achieve good protection. When lesions are not clearing reassessment of diagnosis may be necessary. Therapeutic options include patient applied and office base therapies. Some patients may need repeated cycles of therapy or combined modalities. Counselling for smoking cessation and HIV testing should be offered. High level of anxiety and sexual concerns are common. Partner notification is not recommended. Patients with GW and their actual and future partner(s) should be counselled about HPV quadrivalent prophylactic vaccine.

\section{S13 Respondent-driven sampling: where we are and where should we be going?

S13.1 RESPONDENT-DRIVEN SAMPLING: USES, ASSUMPTIONS,
LIMITS AND PROSPECTS

doi:10.1136/sextrans-2011-050102.52

S Frost. University of Cambridge, Cambridge, UK

Respondent-driven sampling (RDS) is widely used to obtain estimates of quantities such as HIV prevalence, but is also used to examine correlates of infection, and less frequently, to help characterise social networks of individuals in "hidden" populations. The popularity of RDS stems in part from the often rapid recruitment of individuals from the target population as well as from the potential to obtain (asymptotically) unbiased estimates using information only from the sample. I will review the assumptions required for this potential to be realised, whether these assumptions are broken in practice, and the impact of breaking assumptions on statistical inference. I will also discuss improvements to the design and analysis of RDS studies, including schemes for giving out coupons, obtaining absolute sizes of at-risk populations using capture-recapture, and the utility of detailed social network inventories.

\section{S13.2 ASSESSING RESPONDENT-DRIVEN SAMPLING}

doi:10.1136/sextrans-2011-050102.53

S Goel. Yahoo Research, New York, USA

Respondent-driven sampling is a network-based technique for estimating traits in hard-to-reach populations, for example, the prevalence of HIV among drug injectors. In recent years RDS has been used in more than 120 studies in more than 20 countries, and by leading public health organisations, including the Centers for Disease Control and Prevention in the USA. Despite the widespread use and growing popularity of RDS, there has been little empirical validation of the methodology. In this talk, I investigate the performance of RDS by simulating sampling from 85 known, network populations. Across a variety of traits we find that RDS is substantially less accurate than generally acknowledged, and that reported RDS CI are misleadingly narrow. Moreover, it is unlikely RDS performs any better in practice than in our simulations as we model a best-case scenario in which the theoretical RDS sampling assumptions hold exactly. Notably, the poor performance of RDS is driven not by the bias, but by the high variance of estimates, a possibility that had been largely overlooked in the RDS literature. Given the consistency of our results across networks and our generous sampling conditions, we conclude that RDS as currently practiced may not be suitable for key aspects of public health surveillance where it is now extensively applied. This work is joint with Matthew Salganik.

\section{S13.3 AN EMPIRICAL EVALUATION OF RESPONDENT-DRIVEN SAMPLING}

doi:10.1136/sextrans-2011-050102.54

${ }^{1,2} \mathrm{~N}$ McCreesh, ${ }^{3} \mathrm{~S}$ Frost, ${ }^{1,2,4,5} \mathrm{~J}$ Seeley, ${ }^{4} \mathrm{~J}$ Katongole, ${ }^{4} \mathrm{M}$ Ndagire Tarsh, ${ }^{4} \mathrm{R}$ Ndungutse, ${ }^{1,2} \mathrm{~F}$ Jichi, ${ }^{4,1,2} \mathrm{D}$ Maher, ${ }^{6} \mathrm{P}$ Sonnenberg, ${ }^{6} \mathrm{~A}$ Copas, ${ }^{1,2} \mathrm{R}$ J Hayes, ${ }^{1,2} \mathrm{R}$ G White. ${ }^{1}$ Department of Infectious Disease Epidemiology, London School of Hygiene and Tropical Medicine, UK; ${ }^{2}$ Faculty of Epidemiology \& Population Health, London School of Hygiene and Tropical Medicine, UK; ${ }^{3}$ Department of Veterinary Medicine, University of Cambridge, Cambridge, UK; ${ }^{4}$ MRC/UVRI Uganda Research Unit on AIDS, Entebbe, Uganda; ${ }^{5}$ School of International Development, University of East Anglia, Norwich, UK; ${ }^{6}$ Department of Infection and Population Health, UCL, UK

Objective Respondent-driven sampling (RDS) is an increasingly widely used variant of snowball sampling, that proponents claim can provide unbiased estimates. RDS has not been rigorously evaluated in the field. This study evaluated RDS by comparing estimates from an RDS survey with total-population data.

Methods Total-population data on age, tribe, religion, socioeconomic status, sexual activity and HIV status were available on a population of 2402 male household-heads from an open cohort in rural Uganda. An RDS survey was carried out in this population, employing current RDS methods of sampling (RDS-sample) and statistical inference (RDS-estimates). Analyses were repeated for the full RDS sample and a small sample of the first 250 recruits (including 10 seeds). 